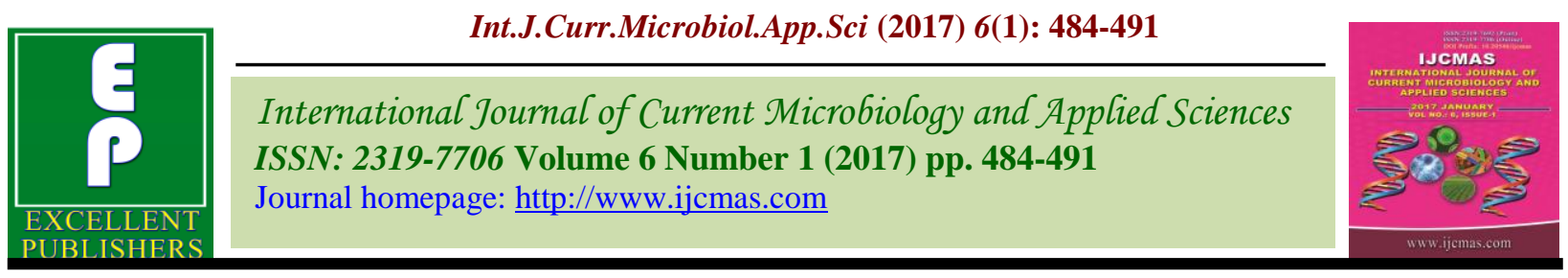

Original Research Article

http://dx.doi.org/10.20546/ijcmas.2017.601.057

\title{
Study the Utility of Cryptococcal Latex Agglutination Test in HIV-Positive and Negative Patients Suspected of having Cryptococcal meningitis and Compare with India Ink Preparation and Fungal Culture
}

\author{
Susheela Chaurasia, Ashwini Waghmare* and Ameeta Joshi \\ Department of Microbiology, Grant Medical College, Mumbai, India \\ *Corresponding author
}

\section{A B S T R A C T}

Keywords

Cryptococcal meningitis, fungal culture, HIV Positive, Cryptococcal antigen, India ink preparation.

Article Info

Accepted:

23 December 2016

Available Online:

10 January 2017
Among the non-tuberculous causes of meningitis, cryptococcal meningitis tops the list with prevalence around five percent in India. The present study was carried out to compare latex agglutination test with India Ink Preparation and fungal culture admitted to a tertiary care hospital. The samples for the study were received from all age groups of HIV-positive patients suspected of having meningitis admitted to either medicine or paediatric ward and HIV-negative patients with signs and symptoms of meningitis were taken as control group. Out of 1,224 HIV-positive patients, 224 were clinically suspected cases of meningitis. Antigen detection test was done on 160 patients irrespective of their HIV status. Of these, 22 cases were found to be positive for the cryptococcal antigen detection test; only 18 cases were culture positive and the remaining four $(2.82 \%)$ were culture negative. Majority $(18,81.8 \%)$ of the HIVpositive patients with cryptococcal meningitis, had $\mathrm{CD}^{+}$T-lymphocyte cell count < 200 cells $/ \mu \mathrm{l}$. It was observed that cryptococcal antigen detection test was more sensitive (100\%) as compared to culture (81.8\%) and India ink preparation $(68.2 \%)$.

\section{Introduction}

Cryptococcosis is a leading mycological cause of morbidity and mortality among AIDS patients. In many patients, cryptococcosis is the first indication of AIDS (Mitchell et al., 1995). Meningitis is the most common manifestation of cryptococcal disease in patients with HIV infection (Koralnik, 2000). Cryptococcosis is the second most common fungal infection after candidiasis in HIV patients. Worldwide, around seven to ten percent of patients with AIDS are affected with cryptococcal infection; most of these cases are sporadic in nature.
In India, pulmonary tuberculosis is the earliest and the most common opportunistic infections in HIV/AIDS patients.

Among the non-tuberculous causes of meningitis, cryptococcal meningitis tops the list with prevalence around five percent in India and five to seven percent in U.S (Koralnik, 2000). The present study was carried out to find out the incidence of cryptococcal meningitis in HIV/AIDS patients and compare latex agglutination test with India Ink Preparation and fungal culture admitted to a tertiary care hospital and to 
correlate these findings with the immunological status $\left(\mathrm{CD}^{+}{ }^{+}\right.$-lymphocyte) of the patients.

The main aim and objectives of this study the utility of cryptococcal latex agglutination test in HIV-positive and negative patients suspected of having cryptococcal meningitis and compare with India Ink Preparation and fungal culture.

\section{Materials and Methods}

This was a prospective study carried out in Department of Microbiology of a tertiary care hospital from October 2007 to May 2009.

Inclusion criteria: The samples for the study were received from all age groups of HIVpositive patients suspected of having meningitis admitted to either medicine or paediatric ward and HIV-negative patients with signs and symptoms of meningitis were taken as control group.

Exclusion criteria: Cases of post-traumatic meningitis and meningitis developing after cranial surgery were excluded from the study.

Detailed clinical history as regards to the age, sex, clinical signs and symptoms, previous history of similar illness, any previous treatment or investigations done and treatment history were obtained from both HIV-positive and HIV-negative groups.

CSF was collected by the clinician by performing lumbar puncture with all aseptic precautions. The sterile bulbs were labelled and transported to the microbiology laboratory immediately. These specimens were processed in the laboratory without any delay. Gross examination: The CSF was observed for colour, turbidity, cobweb and presence of blood and clot.

Microscopic examination: Unstained wet mount, Gram stain, Acid fast stain, India ink preparation (IIP) was done.

Culture of CSF: Bacteriological culture, Fungal culture

Antigen detection test for Cryptococcus neoformans

India ink preparation (Fig.II): This method was used for the identification of encapsulated organisms such as the yeast $C$. neoformans. A loopful of the centrifuged sediment was mixed with India ink on a slide, smear was made and dried. Examined under $10 \mathrm{X}$ and $40 \mathrm{X}$ for the presence of typical encapsulated, spherical, budding yeast forms measuring 4-20 micrometer of $C$. neoformans.

Fungal culture (Chander, 2002): The centrifuged deposit was inoculated onto two Sabouraud Dextrose Agar slants. The media was supplemented with antibiotic chloramphenicol $(0.05 \mathrm{mg} / \mathrm{ml})$ to minimise the bacterial contamination and without cycloheximide since it is inhibitory for the growth of cryptococcus. The slants were incubated at $25^{\circ} \mathrm{C}$ and $37^{\circ} \mathrm{C}$ separately for over a period of seven days with observation every 48 hours.

Growth on SDA (Fig. IV) was subjected to lacto phenol cotton blue (LPCB) mount. Yeast like colonies were identified as per the standard mycological procedures using tests such as germ tube test, Dalmau technique, urease test, carbohydrate fermentation test, carbohydrate assimilation test. Confirmation of the growth of $C$. neoformans (Fig.III) was done by the development of brown colour on bird seed agar (Chander, 2002; Chakrabarti et al., 2008, Sutton et al., 1998).

Latex agglutination test: The supernatant from the centrifuged CSF sample was used for the detection of antigen against $C$. neoformans by latex agglutination test. The kit (Fig.I) was 
provided by Immuno-Mycologics, Inc. The test was performed as per the manufacturers' instruction. Antigen detection test was applied only to those samples which were obtained from clinically suspected cases of cryptococcal meningitis irrespective of their HIV/AIDS status.

Statistical analysis was done by SPSS software version 15 demo. Data was represented in the form of frequency and percentages. Cross tabulation was done to assess association between variables. ChiSquare test was applied with continuity correction test wherever it was applicable. A $p$ value of $<0.05$ was considered to be statistically significant.

\section{Results and Discussion}

A total of 25,897 samples were received by the microbiology department during the study period for HIV testing, of which 14,378 samples were from medicine and paediatric departments. Of these, 1,224 were tested positive for HIV antibodies (as per the NACO HIV testing guidelines).

Out of these 1,224 HIV-positive patients, 224 were clinically suspected cases of meningitis. The clinical diagnosis mentioned in the requisition form was TBM (105 cases i.e. $46.9 \%$ ), cryptococcal meningitis (72 cases i.e. $32.1 \%$ ), pyogenic meningitis (34 cases i.e. $15.2 \%$ ), toxoplasmosis (6 cases i.e. $2.7 \%$ ), viral meningitis (4 cases i.e. 1.8\%) and syphilitic meningitis (3 cases i.e. 1.3\%). A total of 181 cases of meningitis which were negative for HIV antibodies were taken as the control group. All HIV-positive patients were further tested for their CD4 status.

Of the total 22 cases of cryptococcal meningitis, 18 were culture positive in which $14(77.8 \%)$ were males and four $(22.2 \%)$ were females. Majority of the patients $(17,94.4 \%)$ were in the age group 16-45 years.
Antigen detection test was done on 160 patients irrespective of their HIV status. Of these, 22 cases were found to be positive for the cryptococcal antigen detection test; only 18 cases were culture positive and the remaining four $(2.82 \%)$ were culture negative. All the 22 patients were from HIV-positive group. The remaining 138 culture negative cases were found to be negative for the antigen detection test.

All the 15 IIP positive cases were uniformly positive for culture and antigen detection test. In the HIV-negative control group, there were no fungal isolates and all were negative for IIP. Antigen detection test was performed in 12 clinically suspected cases of cryptococcal meningitis and all these cases were found to be negative.

Majority $(18,81.8 \%)$ of the HIV-positive patients with cryptococcal meningitis, had $\mathrm{CD}^{+}$T-lymphocyte cell count $<200$ cells/ $\mu 1$. The remaining four had CD4 cell count in the range $200-499$ cells/ $\mu 1$.

Prevalence of cryptococcal meningitis among AIDS patients has been reported to range from 2-30\%. Cryptococcal meningitis has been relatively understudied in developing countries such as India, while, cryptococcal meningitis has been reported from the Indian subcontinent, showing a high prevalence (Satpute et al., 2006, Jaiswal et al., 2002).

In the present study, all the culture positive fungal isolates were obtained from HIV positive patients. $C$. neoformans was uniformly isolated from all these cases. This indicates that HIV positivity is an important risk factor associated with cryptococcal meningitis. No fungal isolates were obtained from HIV-negative control group. The overall prevalence of cryptococcal meningitis on the basis of culture positivity in HIV/AIDS patients with meningitis was $8 \%$. Prasad et 
al., observed in their study, that, out of the 45 patients, who developed cryptococcal meningitis, no identifiable risk factors were detected in $18(40 \%)$ patients, whereas, in the remaining 27 (60\%) patients, HIV infection was found as a risk factor in the maximum number of patients $(13,28.9 \%)$ (Prasad et al., 2003).

In the present study, India ink preparation was positive in $15(83.3 \%)$ out of the $18(100.0 \%)$ culture positive cases, whereas, it was negative in the remaining three $(16.7 \%)$ culture positive cases. Heyderman et al., in their study found that India ink preparation was positive for $C$. neoformans for 76 (85\%) of 89 patients, CSF culture was positive for 77 (87\%) out of 899 (Heyderman et al., 1998). In a study done by Khanna et al., India ink staining was positive in 13 out of the total 18 (72.2\%) HIV-positive patients whereas, fungal culture was positive in all cases (Khanna et al., 1996). Thus, it is seen from various studies that the sensitivity of India ink preparation is low when compared to CSF culture.

Latex agglutination test for detection of cryptococcal polysaccharide antigen is a highly sensitive, specific and rapid test. It is useful for both in detecting the $C$. neoformans infection as well as, in monitoring the response to therapy. The antigen remains detectable for several months after infection and is, therefore, a suitable choice of laboratory test for screening (Satpute et al., 2006; Goodman et al., 1975; Kaufman et al., 1968). In the present study, a total of 22 cases were found to be positive for antigen detection test. Of these, 18 were also positive for culture. However, in four $(2.82 \%)$ cases, antigen detection test alone was positive. All these four cases showed a high antigen positive titer (one patient- 1:256, two patients-
1:128, one patient- 1:64). The negative culture report of these cases may be attributed to the presence of dead fungi. Thus, the diagnosis of these four cryptococcal meningitis cases was based on clinical history, HIV-positivity, low CD4 count and antigen titer.

In a study done by Capoor et al., antigen detection test was found to be positive in all 13 samples, whereas, culture was positive only in 10 out of the total samples. From their study, the overall positivity for antigen and culture was found to be $70-90 \%$ and $80-92 \%$ respectively. Heyderman et al., in their study observed that $76(85 \%)$ of the 89 patients were positive by culture. Cryptococcal antigen was positive for 79 (92\%) of 86 patients. In six (7\%) cases, testing for India ink and culture was negative, but the cryptococcal antigen was positive. From all the above studies, it is observed that cryptococcal latex agglutination test is more sensitive than culture in detecting $C$. neoformans infection.

Various studies have found that identification of fungus by India ink preparation had a sensitivity of $82 \%$, while, $100 \%$ sensitivity was observed both in positive fungal culture and detection of cryptococcal antigen in serum/CSF (Kovacs et al., 1985; Snow et al., 1975). In the present study, (Table no.1) when culture, India ink preparation and cryptococcal antigen detection tests were compared, it was observed that, out of the 18 cryptococcal culture positive cases, India ink preparation was positive in $15(83.3 \%)$ cases, whereas, cryptococcal antigen detection test was uniformly positive in all these culture positive cases. However, in four $(2.82 \%)$ cases, the cryptococcal antigen detection test alone was positive and culture was negative. 
Table.1 Comparison of the results of the growth of Cryptococcus, Cryptococcal antigen detection test and the India Ink Preparation (IIP) among the HIV positive patients and the HIV negative control group

\begin{tabular}{|c|c|c|c|c|c|}
\hline \multirow{2}{*}{$\begin{array}{c}\text { Culture } \\
\text { result }\end{array}$} & & \multicolumn{2}{|c|}{ IIP (n=405) } & \multicolumn{2}{c|}{$\begin{array}{c}\text { Antigen detection test } \\
\text { (n=160) }\end{array}$} \\
\cline { 2 - 6 } & & IIP positive & IIP negative & $\begin{array}{c}\text { Antigen } \\
\text { positive }\end{array}$ & Antigen negative \\
\hline $\begin{array}{c}\text { Culture } \\
\text { positive }\end{array}$ & No. & 15 & 3 & 18 & 0 \\
\cline { 2 - 6 } & $\%$ & $83.3 \%$ & $16.7 \%$ & $100.00 \%$ & $0.00 \%$ \\
\hline $\begin{array}{c}\text { Culture } \\
\text { negative }\end{array}$ & No. & 0 & 387 & 4 & 138 \\
\cline { 2 - 6 } & $\%$ & $0.0 \%$ & $100.0 \%$ & $2.82 \%$ & $97.18 \%$ \\
\hline Total & No. & 15 & 390 & 22 & 138 \\
\cline { 2 - 6 } & $\%$ & $3.7 \%$ & $96.3 \%$ & $13.75 \%$ & $86.25 \%$ \\
\hline
\end{tabular}

In the HIV-negative control group, there were no fungal isolates and all were negative for IIP. Antigen detection test was performed in 12 clinically suspected cases of cryptococcal meningitis and all these cases were found to be negative.

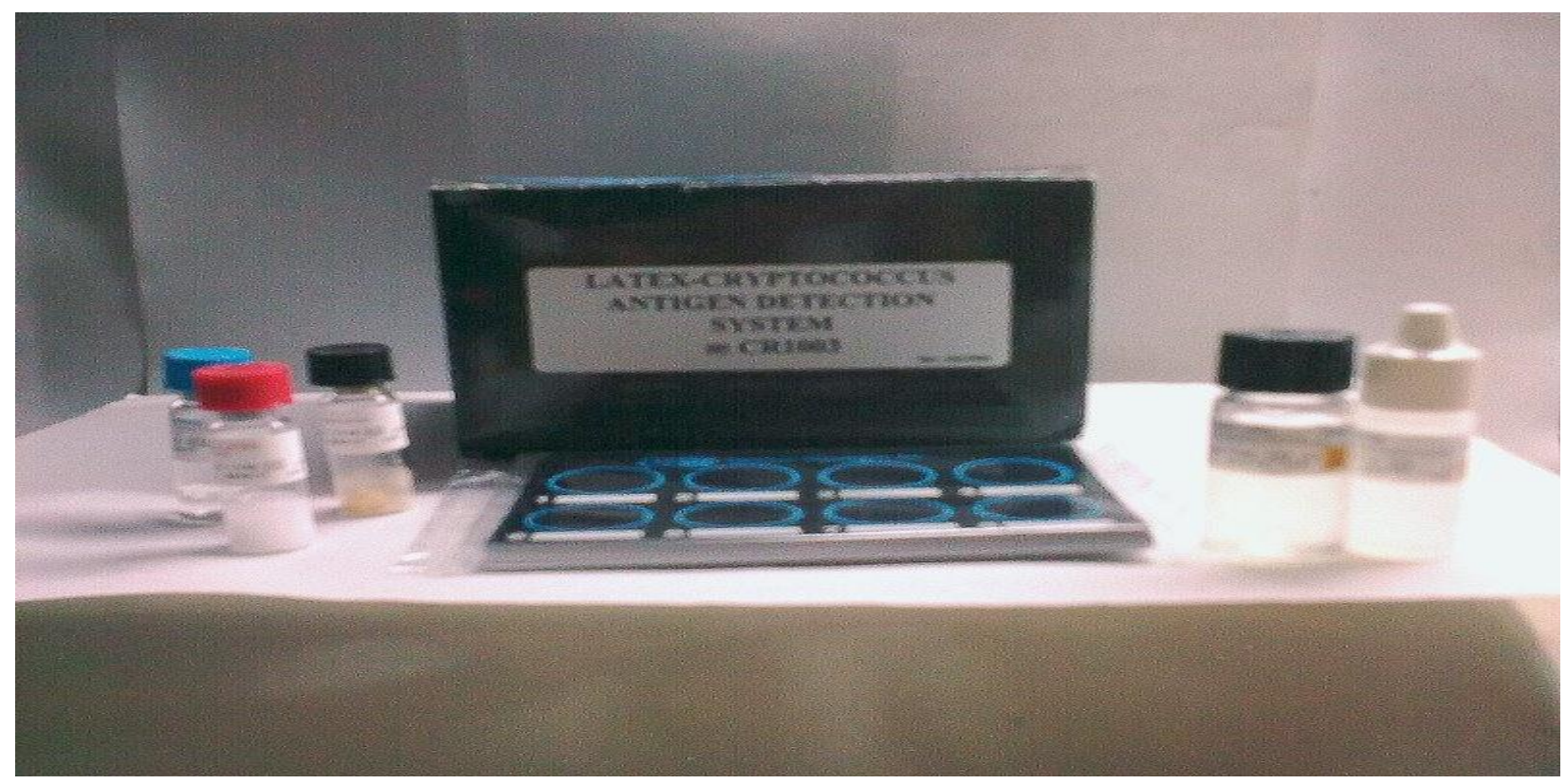

Fig.1 Latex-Cryptococcus Antigen Detection Kit 
Fig.2 India Ink preparation showing encapsulated budding yeast cells of Cryptococcus neoformans

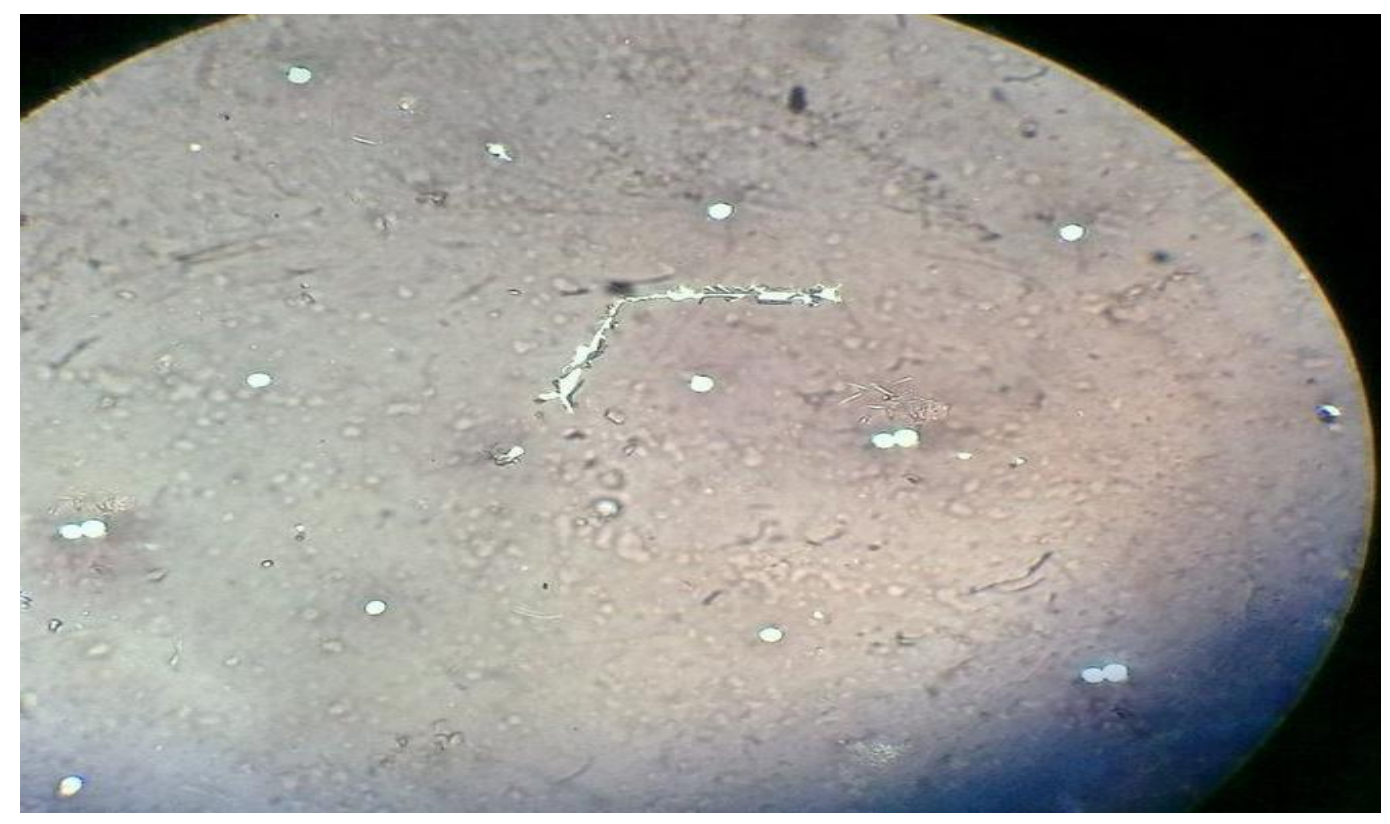

Fig.3 Bird seed agar showing brown coloured colonies of Cryptococcus neoformans

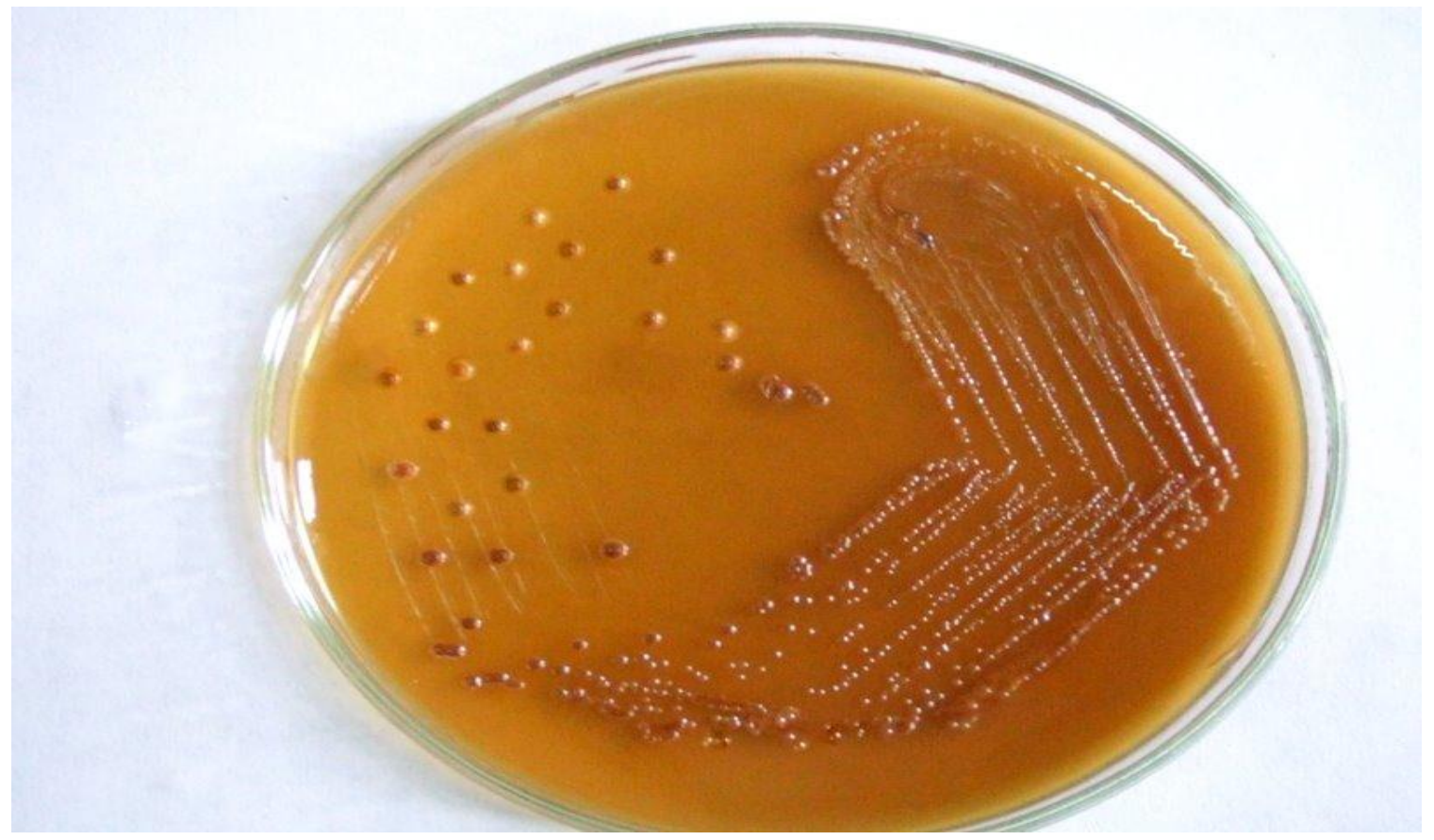


Fig.4 Sabouraud Dextrose Agar showing creamy mucoid colonies of Cryptococcus neoformans

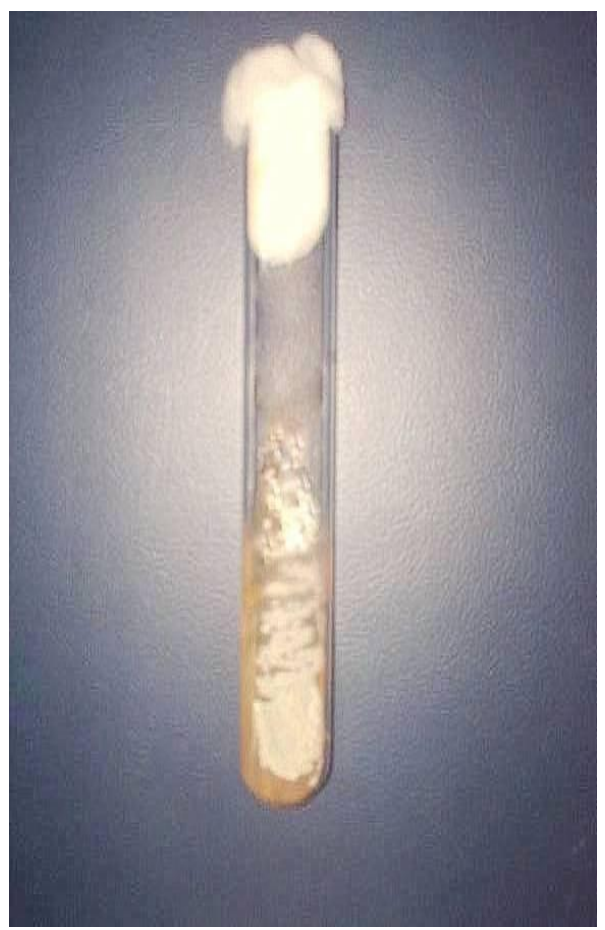

Thus, in this study, cryptococcal antigen detection test was found to be more sensitive for detecting the antigens in the CSF, when compared with culture and India ink preparation. Similar findings were shown by Mitchell et al., with cryptococcal antigen detection test sensitivity (95\%) more than either culture $(75 \%)$ or India ink preparation (50\%). Capoor et al., in their study found the overall positivity of microscopy; culture and latex agglutination test to be $79-90 \%, 80-92 \%$ and $95-100 \%$ respectively. These findings are well comparable with the present study.

In conclusion, all culture positive cases of cryptococcal meningitis showed positive cryptococcal antigen detection test. India ink preparation, cryptococcal antigen detection test and the culture showed a correlation of $83.3 \%$ in cases of cryptococcal meningitis. It was observed that cryptococcal antigen detection test was more sensitive $(100 \%)$ as compared to culture (81.8\%) and India ink preparation $(68.2 \%)$.

\section{Acknowledgement}

The authors would thank the HOD, Department of Microbiology and Dean of Grant Medical College, Mumbai and all the staff of Microbiology department for assisting in the study.

\section{References}

Capoor, M.R., Nair, D., Deb, M., Gupta, B., Aggarwal, P. 2007. Clinical and mycological profile of cryptococcosis in a tertiary care hospital. Ind. J. Med. Microbiol., 25: 401-404. 4.

Chakrabarti, A. and Shivaprakash, M.R. 2008. Medical Mycology Laboratory procedures. Dept. of Microbiology, PGI, Chandigarh.

Chander, J. 2002. Textbook of Medical Mycology. 2 Ed. Mehta publishers, New Delhi.

Goodman, J.S., Kautman, L., and Koenig, M.G. 1975. Diagnosis of cryptococcal meningitis: value of immunologic 
detection of cryptococcal antigen. New

Engl. J. Med., 285: 434-436.

Heyderman, R.S., Gangaidzo, I.T., Hakim, J.G., Mielke, J., Taziwa, A., Musvaire, P. et al. 1998. Cryptococcal Meningitis in Human Immunodeficiency VirusInfected Patients in Harare, Zimbabwe. Clin. Infect. Dis., 26: 234-289.

Jaiswal, S.P., Hemwani, N., Sharma, S., Athale, S., Chitnis, D.S. 2002. Prevalence of fungal meningitis among HIV positive and HIV negative subjects in Indore (MP State). Ind. J. Med. Sci., 56: $325-329$.

Kaufman, L., Blumer, S. 1968. Value and interpretation of serological tests for diagnosis of cryptococcosis. Appl. Microbiol., 16: 1907-1912.

Khanna, N., Chandramukhi, A., Desai, A. and Ravi, V. 1996. Cryptococcal infections of the central nervous system: an analysis of predisposing factors, laboratory findings and outcome in patients from south India with special reference to HIV infection, J. Med. Microbiol., 45: 376-379.

Koralnik, I.J. 2000. Neurologic disease caused by Human Immunodeficiency Virus 1 and Opportunistic infections, Chapter 120. In: Mandell, Douglous and Bennett's Principles and practice of infectious diseases, 6 Ed. Mandell G L, Bennett J E, Dolin R. Eds. Churchill livingstone, Edinburgh, 1583-1601.

Kovacs, J.A., Kovacs, A.A., Polios, M. et al. 1985. Cryptococcosis in Acquired Immunodeficiency Syndrome. Ann. Intern. Med., 103: 533-538.

Mitchell, T.G. and Perfect, J.R. 1995. Cryptococcosis in the era of AIDS-100 years after the discovery of Cryptococcus neoformans. Clin. Microbiol. Rev., 8: 516-535.

Prasad, K.N., Agarwal, J., Nag, V.L., Verma, A.K., Dixit, A.K., Ayyagari, A. 2003. Cryptococcal Infection in patients with clinically diagnosed meningitis in a tertiary care centre. Neurol., 51: 364366.

Satpute, M.G., Telang, N.V., Litake, G.M., Niphadkar, K.B., Joshi, S.G. 2006. Prevalence of cryptococcal meningitis at a tertiary care centre in Western India (1996-2005). J. Med. Microbiol., 55: 1301-1302.

Snow, R.M., Dismukes, W.E. 1975. Cryptococcal meningitis-Diagnostic value of cryptococcal antigen in CSF. Arch. Int. Med., 135: 1155-1157.

Sutton, D.A., Fothergill, A.W., Rinaldi, M.G. 1998. Guide to Clinically Significant Fungi. Williams and Wilkins Baltimore.

\section{How to cite this article:}

Susheela Chaurasia, Ashwini Waghmare and Ameeta Joshi. 2017. Study the Utility of Cryptococcal Latex Agglutination Test in HIV-Positive and Negative Patients Suspected of having Cryptococcal meningitis and Compare with India Ink Preparation and Fungal Culture. Int.J.Curr.Microbiol.App.Sci. 6(1): 484-491. doi: http://dx.doi.org/10.20546/ijcmas.2017.601.057 